\title{
Padrão e necessidades de atendimento pré-hospitalar a idosos
}

\author{
Standard and needs of pre-hospital care for elderly
}

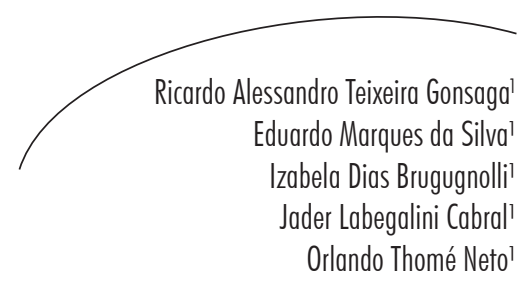

\section{Resumo}

As principais doenças que atingem os idosos são as crônico-degenerativas, que somadas às frequentes comorbidades desse grupo, demandam maior preocupação por parte das instituições de saúde e, consequentemente, maior utilização de serviços de alta complexidade. O objetivo do estudo foi descrever os atendimentos de idosos pelo Serviço de Atendimento Móvel de Urgência (SAMU) no município de Catanduva-SP, com base em registros de atendimentos aos indivíduos com idade superior a 59 anos lá realizados. Foram analisados dados demográficos, sinais vitais, tipos de atendimento segundo gênero, nível de atenção à saúde indicado para resolução da ocorrência, horários das ocorrências e relação entre os grupos etários e ocorrências de clínica médica e traumáticas. Foram atendidos 42.629 pacientes, a maioria do sexo feminino (55\%); a média de idade foi de 74,2 anos. Houve significância estatística nos sinais vitais quando comparados os gêneros. As ocorrências de maior frequência foram as classificadas como enfermidades clínicas, seguidas por solicitações sociais e transferências intrahospitalares. Houve aumento dos agravos agudos clínicos e traumáticos nas faixas etárias de maior idade, e 77,9\% dos atendimentos necessitaram de uma unidade fixa de atendimento para continuidade do tratamento. Encontrou-se, ainda, predomínio de atendimento nos horários de vigília dos pacientes. Observam-se maiores taxas de ocorrências com o aumento da faixa etária e utilização mais frequente de atendimento hospitalar terciário pós-ocorrências, fato que caracteriza, de maneira geral, o modelo hospitalocêntrico.

Palavras-chave: Assistência Pré-Hospitalar. Medicina de Emergência. Epidemiologia Descritiva. Serviços Médicos de Emergência. Idoso.

Faculdades Integradas Padre Albino, Curso de Medicina. Catanduva, SP, Brasil. 


\section{Abstract}

The main diseases affecting the elderly are chronic degenerative, which added to the frequent comorbidity of this group, require greater concern on the part of health care institutions and greater use of high complexity services. This study aimed to describe the care of the elderly by the Mobile Emergency Service (SAMU) in the municipality of Catanduva-SP, Brazil, based on attendance records to individuals older than 59 years there performed. We analyzed demographic data, vital signs, according to gender service types, level of health care indicated for resolution of the occurrence of events and schedules relationship between age groups and medical and clinical traumatic occurrences; 42,629 patients were seen, most women (55\%); the average age was 74.2 years. There were significant differences in vital signs when comparing genders. Most frequent occurrences were classified as the clinical disease, followed by social demands and in-hospital transfers. There was an increase of clinical acute and traumatic injuries in older age groups, and $77.9 \%$ of visits required a fixed unit of service for continuing care. It was also found predominance of patients during waking hours. We observed higher rates of events with increasing age and increased use of tertiary hospital care after-occurrences, which is characteristic, in general, of the hospital-centered model.
Key words: Prehospital Care. Emergency Medicine. Epidemiology, Descriptive. Emergency Medical Services. Elderly.

\section{INTRODUÇão}

Nos indivíduos idosos, grande parte da morbimortalidade se concentra em doenças crônicodegenerativas, em geral associadas em quadros de comorbidade que demandam assistência de alta complexidade. ${ }^{1}$ Dados do Ministério da Saúde (DataSUS, 2012) ${ }^{2}$ revelam que a principal causa de morte dos pacientes acima de 60 anos são as doenças cardiovasculares, seguidas pelas neoplásicas, estando as causas externas na terceira posição.

As estatísticas sobre as causas de mortalidade mostram que os acidentes e violências afetam predominantemente a população jovem e adulta; entretanto, as causas externas também merecem atenção entre as pessoas idosas. As taxas de mortalidade mostram-se crescentes à medida que avança a idade, possivelmente pela fragilidade adquirida com o ganho de mais anos de vida. ${ }^{3}$ Existem poucos estudos na literatura nacional e internacional sobre o atendimento ao idoso, com exceção dos pacientes vitimados de causas externas. A maioria versa sobre a priorização no atendimento, problemas cardiovasculares e modelos teóricos de eixos de assistência. ${ }^{4-8}$ Os idosos vítimas de trauma chegam mais graves ao hospital e consomem mais recursos no tratamento., ${ }^{9,10}$ As alterações estruturais e funcionais próprias do envelhecimento, associadas à multimorbidade, predispõem os idosos a diversos acidentes e explicam as diferenças básicas quanto ao espectro das lesões, a dominância entre os gêneros, a duração e a evolução esperada. ${ }^{11}$

Este artigo em por objetivo caracterizar o perfil do paciente idoso atendido por um serviço de atendimento móvel de urgência (préhospitalar).

\section{METODOLOGIA}

Trata-se de estudo transversal, realizado com dados da central de regulação do SAMU de Catanduva-SP, que abrange a região administrativa de Catanduva (18 municípios). Foram analisados os dados populacionais (gênero e idade completa 
no momento do atendimento), os sinais vitais no momento do atendimento, o motivo da solicitação de atendimento, tipo de atendimento local (equipe da unidade de suporte básico - USB e da unidade de suporte avançado - USA), nível de atenção à saúde indicado para a resolução da ocorrência e horário das ocorrências dos pacientes com idade igual ou superior de 60 anos.

Os dados foram coletados entre janeiro de 2006 e julho de 2012, totalizando 42.629 pacientes. Foram avaliadas a relação entre a proporção da faixa etária pela população total (variável populacional - A) e a proporção das ocorrências classificadas como clínica médica com relação aos atendimentos totais por faixa etária (variável Clínica Médica - B), e a proporção das ocorrências classificadas como trauma com relação aos atendimentos totais por faixa etária (variável trauma $-\mathrm{C}$ ).

A variável A foi encontrada pela relação simples entre os números de indivíduos correspondentes a faixa e população total exposta ao risco de adoecer (pacientes acima de 59 anos), segundo dados da IBGE, através do Censo Demográfico de 2010. As variáveis B e C foram construídas pela relação simples entre o número de ocorrências (clínica para B e trauma para C) e a população exposta ao risco de adoecer.

A análise foi feita usando o software EpiInfo $\AA$ versão 3.5.3. Os dados quantitativos foram expressos em média e desvio padrão, e a análise estatística utilizada foi teste $t$ de Student para amostras independentes com nível de significância de 5\%. As variáveis qualitativas foram expressas em números absolutos e relativos.

As idades foram categorizadas em faixas de cinco anos; os tipos de ocorrências considerados como social foram aqueles em que todo tipo de transporte de pacientes que necessitaram de viaturas simples (tipo $\mathrm{A}$, desprovida de equipe de atendimento pré-hospitalar) para retorno da unidade de atendimento hospitalar fixa para seu domicílio. Nos pacientes classificados como verificação de óbito domiciliar, foram incluídos os que morreram em casa por causas naturais.

Foram classificados como atendimentos de clínica médica os pacientes com queixas e sintomas cardiovasculares (crise hipertensiva, arritmias, hipotensões, entre outros), pneumopatias (dispneia, asma, doença pulmonar obstrutiva crônica, tosse, entre outros), nefropatias (insuficiências renais aguada e crônica, nefrolitíase, infecção do trato urinário, entre outros), neuropatias (acidentes vasculares encefálicos, crises convulsivas, cefaleias, entre outros), endocrinopatias (hiperglicêmicas, hipoglicemias, crises tireotóxicas, entre outras) e demais doenças correlatas. Consideraramse afecções cirúrgicas os pacientes portadores de doenças com tratamento preferencialmente cirúrgico (aneurismas, abdomens agudos, escaras, entre outros).

A rede hierarquizada do Sistema Único de Saúde (SUS) do município de CatanduvaSP apresenta atendimento público e rede suplementar (contratada), incluindo nesta última as unidades filantrópicas e privadas. Diante do banco de dados, não foi possível categorizar doenças pelo código internacional de doenças.

Os sinais vitais no momento do atendimento estudados foram: frequência cardíaca, de pulso e respiratória, as pressões arteriais sistólica e diastólica, temperatura axilar e saturação de hemoglobina em ar ambiente. Foi utilizada a escala de coma de Glasgow para caracterizar a resposta neurológica no momento do atendimento.

O projeto de pesquisa foi aprovado pelo Comitê de Ética em Pesquisa (CEP) das Faculdades Integradas Padre Albino -(FIPA), do curso de Medicina registrado sob o protocolo CAAE 0015.0.218.000-09. 


\section{RESULTADOS}

Foram atendidos 42.629 no período analisado, sendo $55,5 \%$ do gênero feminino $(23.657$ pacientes). A média de idade foi de 74,2 $\pm 9,2$ anos

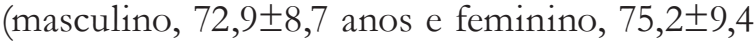
anos), sendo a diferença de atendimentos do gênero feminino estatisticamente significativa $(\mathrm{p}<0,001)$. A tabela 1 representa os sinais vitais discriminados por gênero.

Tabela 1. Comparação das médias das variáveis fisiológicas com o gênero. Catanduva-SP, 2012.

\begin{tabular}{lllll}
\hline Variável & Geral & Masculino & Feminino & $\mathrm{p}$ \\
Frequência cardíaca (bat/min) & $88,8 \pm 30,7$ & $85,1 \pm 27,3$ & $92,5 \pm 33,6$ & $<0,000001$ \\
Frequência respiratória (inc/min) & $21,5 \pm 4,9$ & $21,6 \pm 4,9$ & $21,5 \pm 4,8$ & $>0,999999$ \\
Escala de coma de Glasgow & $14,6 \pm 1,4$ & $14,5 \pm 1,5$ & $14,6 \pm 1,4$ & $>0,999999$ \\
Pressão arterial sistólica (mmHg) & $140,8 \pm 34,9$ & $137,9 \pm 34,0$ & $143,2 \pm 35,6$ & $<0,000001$ \\
Pressão arterial diastólica (mmHg) & $84,4 \pm 18,5$ & $83,4 \pm 18,5$ & $85,3 \pm 18,5$ & $<0,000001$ \\
Frequência de pulso (bat/min) & $89,5 \pm 21,7$ & $88,0 \pm 22,3$ & $89,9 \pm 21,2$ & $<0,000001$ \\
Temperatura $\left({ }^{\circ} \mathrm{C}\right)$ & $36,8 \pm 2,8$ & $36,8 \pm 2,2$ & $36,7 \pm 3,2$ & $>0,999999$ \\
Saturação $(\%)$ & $94,3 \pm 5,8$ & $94,3 \pm 6,2$ & $94,3 \pm 5,5$ & $>0,999999$ \\
\hline
\end{tabular}

Teste $t$ de Student para amostras independentes com intervalo de confiança de 5\%;

Quanto ao tipo de ocorrência (tabela 2), os mais frequentes foram os atendimentos classificados como clínica médica $(45,7 \%)$ em ambos os gêneros, seguidos por solicitações sociais $(23,2 \%)$ e transferências inter-hospitalares $(16,2 \%)$.

Tabela 2. Tipo de ocorrência e gênero do usuário. Catanduva-SP, 2012.

\begin{tabular}{lcccccc}
\hline \multirow{2}{*}{ Tipo de ocorrência } & Feminino & \multicolumn{3}{c}{ Masculino } & \multicolumn{3}{c}{ Total } \\
\cline { 2 - 7 } & $\mathrm{n}$ & $\%$ & $\mathrm{n}$ & $\%$ & $\mathrm{n}$ & $\%$ \\
\hline Clínica médica & 10.502 & 45,7 & 8.988 & 53,9 & 19.490 & 45,7 \\
Clínica cirúrgica & 140 & 60,9 & 103 & 39,1 & 243 & 0,6 \\
Trauma & 2.119 & 58,2 & 1.521 & 41,8 & 3.640 & 8,5 \\
Psiquiatria & 54 & 45,7 & 64 & 54,3 & 118 & 0,3 \\
Constatação de óbito & 314 & 53,9 & 268 & 46,1 & 582 & 1,3 \\
Transferência intra-hospitalar & 3.663 & 53,0 & 3.245 & 47,0 & 6.908 & 16,2 \\
Orientação & 408 & 35,3 & 748 & 64,7 & 1.156 & 2,7 \\
Social & 6.068 & 61,2 & 3.843 & 38,8 & 9.911 & 23,2 \\
Não informado & 153 & 54,4 & 128 & 45,6 & 281 & 0,6 \\
\hline Total & 23.421 & 54,9 & 19.208 & 45,1 & 42.629 & 100,0 \\
\hline
\end{tabular}


Quando analisadas as variáveis A (relação da população da faixa etária com relação à população total exposta), B e C (relação entre o atendimento de clínica e trauma, respectivamente, e a população exposta), observa-se que nas faixas etárias de maior idade, as taxas de agravos agudos clínicos e traumáticos aumentam significativamente ( $p=0,003$ e 0,001, respectivamente), com pico de incidência para a faixa etária acima de 80 anos.

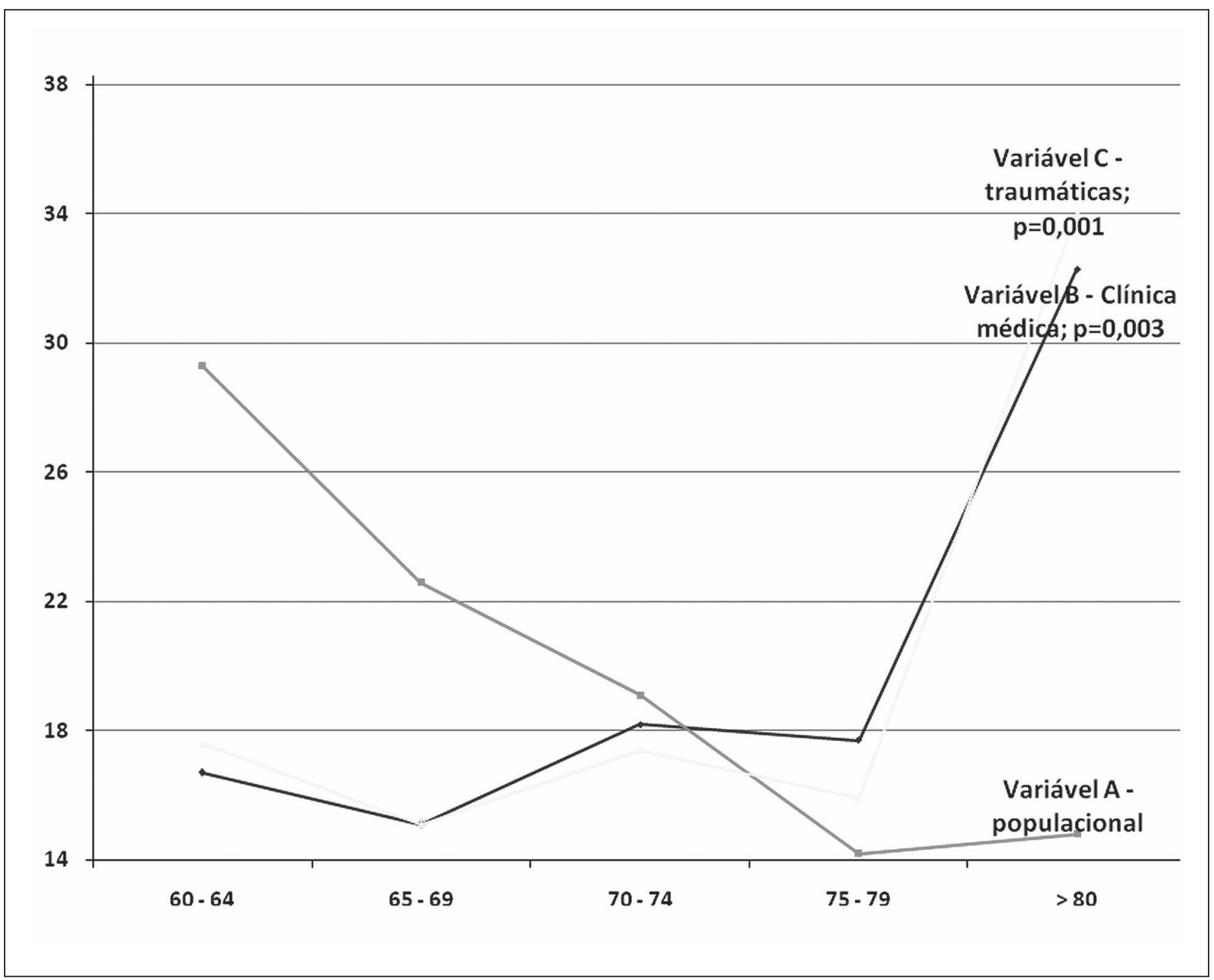

Gráfico 1. Relação entre as variáveis A (populacional), B (ocorrências de clínica médica por faixa etária) e C (ocorrências traumáticas por faixa etária). Catanduva-SP, 2012. 
Os níveis de atenção à saúde indicados para a resolução das ocorrências atendidas são apresentados na tabela 3. O nível terciário foi o mais procurado, seguido pelo primário. Observou-se que $77,02 \%$ dos pacientes foram encaminhados para unidades de prontoatendimento fixas e $14,4 \%$ para o domicílio. Quando analisada a rede de atenção à saúde do município, $76,5 \%$ dos pacientes foram removidos para a unidade de atendimento terciário (23.639 atendimentos), ao passo que $23,5 \%$ deram entrada nas unidades básicas de saúde ou de prontoatendimento de baixa complexidade (7.218 atendimentos). No período, foram constatados 787 óbitos no total, sendo $582 \mathrm{em}$ domicílio e 205 durante o transporte ao hospital.

Tabela 3. Destino dos pacientes atendidos e distribuição na rede hierarquizada. Catanduva-SP, 2012.

\begin{tabular}{|c|c|c|c|}
\hline Destino dos pacientes atendidos & & Frequência & $\%$ \\
\hline Liberados no local & & 81 & 0,2 \\
\hline Recusa de atendimento & & 1.095 & 2,6 \\
\hline Transporte social (domicílio) & & 6.152 & 14,4 \\
\hline Dados não registrados-perdas & & 1.305 & 3,1 \\
\hline Óbitos totais & & 787 & 1,8 \\
\hline Não informado & & 2.302 & 5,40 \\
\hline \multirow[t]{2}{*}{ Rede hierarquizada } & & 30.907 & 77,9 \\
\hline & Total & 42.629 & 100,0 \\
\hline \multirow{3}{*}{$\begin{array}{l}\text { Encaminhamentos dos pacientes segundo nível de } \\
\text { atenção necessário }\end{array}$} & Primária & 5.876 & 19,0 \\
\hline & Secundária & 1.392 & 4,5 \\
\hline & Terciária & 23.639 & 76,5 \\
\hline
\end{tabular}


O gráfico 2 revela quais foram os horários de maior requisição do serviço pré-hospitalar (em intervalos de 15 minutos). De maneira geral, a maioria das chamadas foi no período diurno, com pico às $09 \mathrm{~h} 45$. Vale ressaltar que um número expressivo de chamadas se deu das 7 h15 às $12 \mathrm{~h}$.

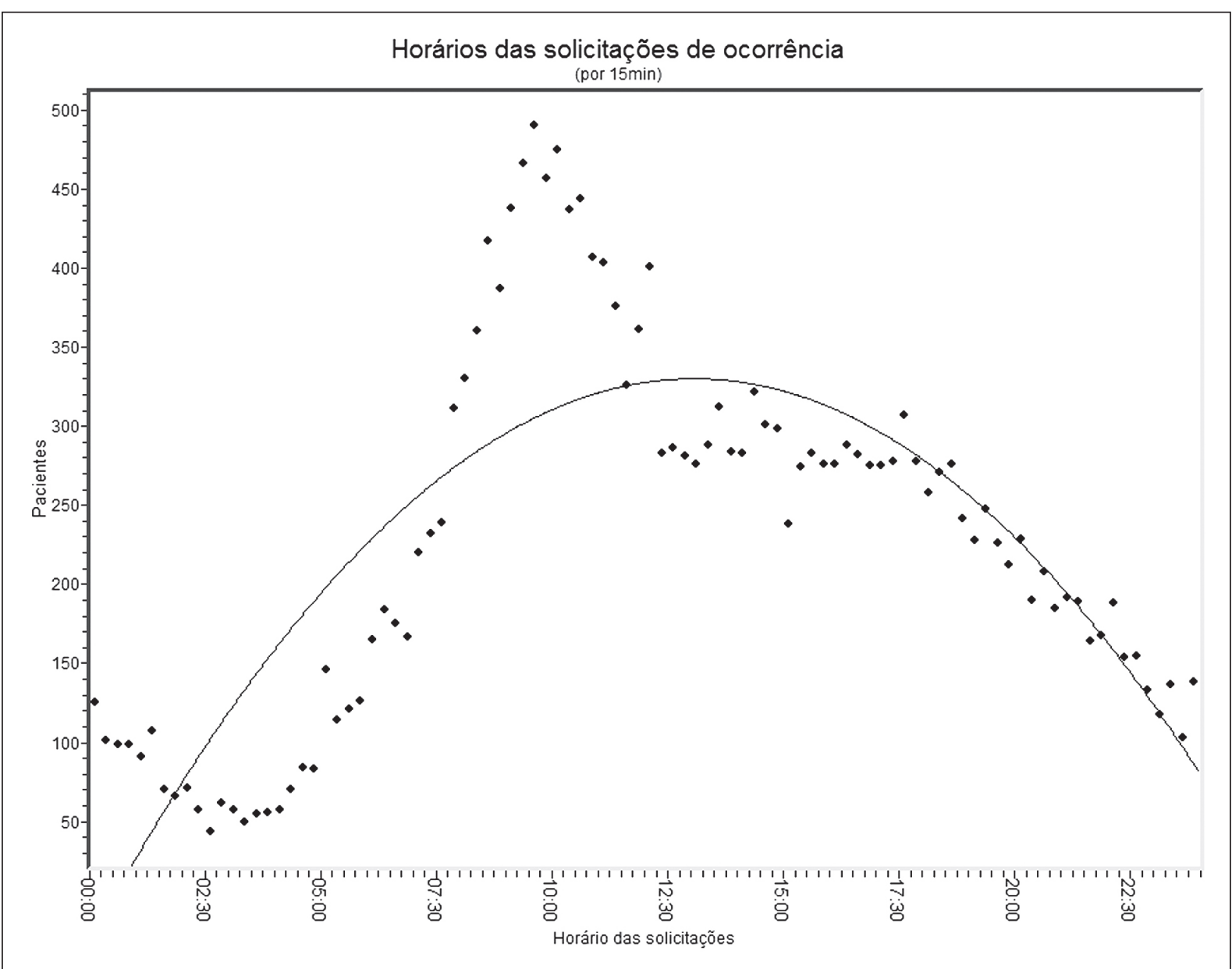

Gráfico 2. Horário de atendimento das ocorrências com pacientes acima de 59 anos do SAMU-192 Catanduva-SP, 2012. 


\section{DISCUSSÃO}

Estudos sobre a maneira como os serviços de saúde são usados por pacientes têm metodologias muito variáveis: dependem da população estudada, do serviço de saúde pesquisado e também de questões socioculturais. No caso deste estudo, a região administrativa de Catanduva-SP (276 mil habitantes, segundo IBGE, 2010) possui uma porcentagem de idosos de $14,6 \%$, acima da média nacional $(10,8 \%)$ e uma renda per capita anual de R\$1.215,40 (SEADE, 2012).

A adoção de formas de vida mais saudáveis propicia o aumento do número de indivíduos idosos e, consequentemente, do risco de eventos agudos associados a doenças crônicas, além da agudização destas. ${ }^{11}$ Segundo Carpenter, ${ }^{5}$ para garantir um atendimento de alta qualidade e eficiência aos pacientes idosos nos Estados Unidos, deverão ser desenvolvidas estratégias de gestões alternativas, tais como melhora dos cuidados pré-hospitalares. Para tanto, é necessário desenvolver novos métodos para a detecção precoce da agudização de doença grave, de modo a orientar o tratamento específico em melhores condições clínicas, realizar avaliações domiciliares periódicas e encaminhar os idosos de alto risco. No entanto, o problema não é de solução simples.

Um programa-piloto na Austrália, com o objetivo de diminuir o número de quedas, com envolvimento de vários atores incluindo o serviço de atendimento pré-hospitalar, não teve o resultado esperado. ${ }^{6}$ Estudos desenvolvidos no estado de Minas Gerais demonstraram que a presença de políticas públicas de Estratégia Saúde da Família e SAMU não encontraram diferenças de morbi-mortalidade para acidente vascular encefálico e infarto agudo do miocárdio na população assistida por essas políticas de saúde pública. ${ }^{2}$ Deve-se também levar em consideração as questões éticas.

No artigo publicado por Fontes \& Pereira, ${ }^{3}$ pesquisando as preferências de atendimento em uma unidade de urgência, observou-se que as escolhas priorizam crianças, jovens e mulheres casadas, e que as tomadas de decisões invocam os princípios éticos de vulnerabilidade, utilidade social e equidade, em detrimento do atendimento geriátrico. Se as múltiplas necessidades dos idosos forem abordadas adequadamente no atendimento pré-hospitalar, a redução de reinternações e o aumento da capacidade funcional podem ser alcançados em médio e longo prazo. A formação integral da equipe de atendimento é necessária para capacitá-la no que diz respeito às particularidades e necessidades da população idosa. ${ }^{12,13}$

Analisando as variáveis fisiológicas pesquisadas (frequência respiratória, frequência cardíaca, escala de coma de Glasgow, pressão arterial sistólica e diastólica e frequência de pulso), pode-se observar que os idosos do sexo feminino têm pressões arteriais e frequências cardíacas ligeiramente maiores do que sexo masculino $(p<0,05)$. Não se observaram diferenças estatísticas com relação a temperatura e saturação de oxigênio pela hemoglobina ( $\mathrm{p}=0,192$ e $\mathrm{p}=0,961$, respectivamente).

Quanto ao motivo da chamada ao serviço de atendimento pré-hospitalar, observou-se predominância de atendimentos clínicos, sociais e transferências inter-hospitalares. Estudos desenvolvidos em Palmas-TO demonstraram que as causas externas são o principal motivo de acionamento do sistema, seguidas por trauma, e que os óbitos são muito mais frequentes nos pacientes acima de 60 anos. ${ }^{14}$

Em estudo na cidade de Porto Alegre-RS, encontrou-se uma taxa de aproximadamente $42 \%$ de atendimentos gerados pelos pacientes acima de 60 anos, quando analisados os agravos clínicos. $^{12}$ Uma discussão importante se faz quanto à utilização das viaturas do atendimento pré-hospital para atendimentos sociais. A utilização do SAMU para o atendimento desta parcela de atenção social é discutível, pois suas viaturas e estruturas devem estar direcionadas na totalidade para o atendimento aos agravos agudos à saúde. Por outro lado, são exceções os municípios que possuem uma rede de assistência social adequada, incluindo o transporte dos usuários idosos e com necessidades especiais. ${ }^{13}$ 
Muitas questões e implicações éticas e legais podem ser suscitadas, tais como a universalidade, equidade e a integralidade da assistência, os princípios da benevolência, não maleficência e justiça, uma vez que os usuários não podem ficar desassistidos, seja nas instituições hospitalares ou no seu ambiente domiciliar.

Conforme demonstrado no gráfico 1 , houve diminuição da proporção dos grupos etários conforme aumentava a idade do paciente (variável A), ao passo que existe aumento relativo significativo dos tipos de ocorrências clínica e traumática (variáveis B e C) com pico após os 80 anos. Assim, as faixas etárias mais avançadas estão mais sujeitas a agravos agudos traumáticos e clínicos, quando comparadas proporcionalmente a outras faixas etárias. Conclusões semelhantes foram encontradas por pesquisadores na cidade de Dallas-EUA, ${ }^{14}$ porém com discrepância na variável "trauma", que diverge dos dados encontrados neste estudo.

O atendimento aos agravos agudos à saúde representa a sobrecarga nas portas das urgências de maior complexidade. ${ }^{15,16}$ Observou-se, ainda, que mais de $76,5 \%$ dos pacientes são encaminhados aos estabelecimentos terciários, em detrimento da rede básica de saúde, agravando as unidades de urgências com o predomínio de pacientes com baixa gravidade. Isso denota falta de adesão e respeito aos protocolos predefinidos com relação a regulação médica e portas de entrada da rede integrada de saúde..$^{17,18}$

O horário dos chamados apresenta um pico por volta das $10 \mathrm{~h}$, com queda seguida por platô que dura até as 20 horas. Observa-se concordância com o período de maior atividade vigil. Não existem fontes confiáveis de comparação na literatura.

A literatura nacional e internacional prendese muito ao estudo das causas externas (violência e traumatismo em geral), em detrimento dos agravos clínicos. Diversos autores relatam a situação de vulnerabilidade a violência e agravos traumáticos, ${ }^{3,7,13,16,18}$ ora chamando a atenção para os dados epidemiológicos, ora para as particulares no atendimento desta população específica. ${ }^{19-22}$ Há autores que afirmam que o trauma no idoso é uma epidemia dos novos tempos, ${ }^{9}$ sendo reconhecidamente importante sua contribuição na mortalidade senil. ${ }^{23}$ Nos dados encontrados neste estudo, observa-se aumento exponencial do número de traumas com relação às faixas etárias, muito mais frequentes ao final da vida.

Ressalta-se que este estudo apresenta limitações com relação ao nível da validade interna, uma vez que foi analisada uma população representativa de uma faixa etária com limitação geográfica. Entretanto, o estudo comprova que essa realidade é transferível a outras regiões do território brasileiro.

Quanto às limitações de nível externo, as variáveis estranhas não podem ser controladas na medida em que a temática abordada é influenciada por aspectos subjetivos como cultura, educação, sentimentos, emoções, crenças e valores - os quais influenciam o atendimento dos pacientes. A validade externa também está condicionada, pois não é possível generalizar locais ou amostras que não as estudadas, permitindo-se apenas a transferência de conclusões para realidades semelhantes. ${ }^{23}$

\section{CONCLUSÃO}

O estudo permite concluir que os sinais vitais apresentam maiores medidas nas idosas, com exceção da saturação de hemoglobina e da temperatura axilar. $\mathrm{O}$ motivo do acionamento do atendimento pré-hospitalar foram os agravos agudos por medicina interna (clínica médica), seguidos por transportes sociais e transferências inter-hospitalares.

As faixas etárias finais da vida apresentam, proporcionalmente, maiores taxas relativas de agravos por indivíduos. Houve predomínio de atendimento hospitalar terciário após atendimento pré-hospitalar, demonstrando a forte tendência hospitalocêntrica; o horário de acionamento foi compatível com o ciclo circadiano (vigil). 


\section{REFERÊNCIAS}

1. Luz CC, Junger WL, Cavalini LT. Análise da atenção pré-hospitalar ao acidente vascular cerebral e ao infarto agudo do miocárdio na população idosa de Minas Gerais. AMB Rev Assoc Med Bras 2010;56(4):452-7.

2. Departamento de Informático do SUS - Datasus [homepage na internet]. C4 mortalidade proporcional por grupo de causas [acesso em 10 de fevereiro de 2015]. Disponível em: tabnet.datasus.gov.br/CGI/ deftohtm.exe?idb2012/c04.def

3. Fortes PAC, Pereira PCA. Priorização de pacientes em emergências médicas: uma análise ética. AMB Rev Assoc Med Bras 2012;58(3):335-40.

4. Comans TA, Currin ML, Quinn J, Tippett V, Rogers A, Haines TP. Problems with a great idea: referral by prehospital emergency services to a community-based falls-prevention service. Inj Prev 2013;19(2):134-8.

5. Carpenter CR, Platts-Mills TF. Evolving prehospital, emergency department, and "inpatient" management models for geriatric emergencies. Clin Geriatr Med 2013;29(1):31-47.

6. Young L, Ahmad H. Trauma in the elderly: a new epidemic? Aust N Z J Surg 1999;69:584-6.

7. Chang TT, Schecter WP. Injury in the elderly and endof-life decisions. Surg Clin N Am 2007;87(1):229-45.

8. Souza JAG, Iglesias ACRG. Trauma no idoso. AMB Rev Assoc Med Bras 2002;48(1):79-86.

9. Melby V, Ryan A. Caring for older people in prehospital emergency care: can nurses make a difference? J Clin Nurs 2005;14(9):1141-50.

10. Ameida NM Neta, Gomes MA, Kanashiro CA, Miranda CC, Paiva AMG, Sampaio DL, et al. Análise do ínidce de amplificação sistólico em pacientes acimas de 55 anos utilizando atenolol associado ou não a diurético. Rev Bras Cardiol 2012;25(1):35-40.

11. Pitteri JSM, Monteiro PS. Caracterização do Serviço de Atendimento Móvel de Urgência (SAMU) em Palmas- Tocantins, Brasil, em 2009. Comun Ciênc Saúde 2010;21(3):227-36.

12. Marques GQ, Lima MAS, Ciconet RM. Agravos clínicos atendidos pelo Serviço de Atendimento
Móvel de Urgência (SAMU) de Porto Alegre - RS. Acta Paul Enferm 2011;24(2):185-91.

13. Gonsaga RAT, Brugugnolli ID, Zanutto TA, Gilioli JP, Silva LFC, Fraga GP. Características dos atendimentos realizados pelo Serviço de Atendimento Móvel de Urgência no município de Catanduva, Estado de São Paulo, Brasil, 2006 a 2012. Epidemiol Serv Saúde 2013;22(2):317-24.

14. Spaite DW, Criss EA, Valenzuela TD, Meislin HW, Ross J. Geriatric Injury: an analysis of prehospital demographics, mechanisms and patterns. Ann Emerg Med 1990;19(12):1418-21.

15. Machado CV, Salvador FGF, O’Dwyer G. Serviço de Atendimento Móvel de Urgência: análise da política brasileira. Rev Saúde Pública 2011;45(1):519-28.

16. Gonsaga RAT, Brugugnolli ID, Fraga FG. Comparison between two mobile pre-hospital care services for trauma patients. World J Emerg Surg 2012;7(Suppl 1):1-8.

17. Thomaz RR, Lima FV. Considerações especiais no atendimento pré-hospitalar ao idoso vítima de trauma. Acta Paul Enferm 2004;17(2):229-34.

18. Souza ER, Correia BSC. Construção de indicadores avaliativos de políticas de atenção à saúde de pessoa idosa vítima de acidentes e violência. Ciênc Saúde Coletiva 2010;15(6):2753-62.

19. Hirano ES, Fraga GP, Montovani M. Trauma no idoso. Medicina (Ribeirão Preto) 2007;40(3):352-7.

20. Lewis MC, Abouelenin K, Paniagua M. Geriatric Trauma: special considerations in the anesthetic management of the injured elderly patient. Anesthesiol Clin 2007;25(1):75-90.

21. Gillies D. Elderly trauma: they are different. Aust Crit Care 1999;12(1):24-30.

22. Deslandes SF, Souza ER. Atendimento préhospitalar ao idoso vítima. Ciênc Saúde Coletiva 2010;15(6):2775-86.

23. Almeida LD, Machado MC. Atitude médica e autonomia do doente vulnerável. Rev Bioét (Impr.) 2010;18(1):165-83. 